\title{
THE NORMAL HOLONOMY GROUP
}

\author{
CARLOS OLMOS
}

(Communicated by Jonathan M. Rosenberg)

\begin{abstract}
We prove that the restricted normal holonomy group of a submanifold of a space of constant curvature is compact and that the nontrivial part of its representation on the normal space is the isotropy representation of a semisimple Riemannian symmetric space.
\end{abstract}

\section{INTRODUCTION}

The restricted holonomy group of a Riemannian manifold is a compact Lie group, and its representation on the tangent space is a product of irreducible representations and a trivial one. This product is unique up to order (see, e.g., [K-N, §5]). Each one of the nontrivial factors is either an orthogonal representation of a connected compact Lie group which acts transitively on the unit sphere or it is the isotropy representation of a simple Riemannian symmetric space of rank $\geq 2$ (see $[B, S])$.

We prove that, surprisingly, all these properties are also true for the representation on the normal space of the restricted normal holonomy group of any submanifold of a space of constant curvature. Moreover, the nontrivial part of this representation is the isotropy representation of a semisimple Riemannian symmetric space.

In order to prove this fact we define a tensor

$$
\mathscr{R}^{\perp}: C^{\infty}(N,(M))^{3} \rightarrow C^{\infty}(N(M)),
$$

which provides the same geometric information as the normal curvature tensor $R^{\perp}$ and has the algebraic properties of a Riemannian curvature tensor. The methods used here are then a slight modification of those of Simons in [S].

\section{NORMAL CURVATURE}

Let $\left(M^{n},\langle\rangle,\right)$ be a Riemannian connected manifold and let $i: M^{n} \rightarrow Q^{N}$ be an isometric immersion, where $Q^{N}$ is of constant curvature.

Received by the editors August 3, 1989 and, in revised form, October 13, 1989.

1980 Mathematics Subject Classification (1985 Revision). Primary 53C40; Secondary 53C35.

Key words and phrases. Normal connection, holonomy group, normal curvature tensor.

This research was supported by International Centre for Theoretical Physics, Trieste (Italy)Universidad Nacional de Cordoba, Cordoba, Argentina. 
Let $N(M) \stackrel{\pi}{\longrightarrow} M$ be the normal bundle over $M$ induced by $i$. For the sake of simplicity the Riemannian metric on $Q^{N}$, as well as the usual metric on the fibers of $N(M)$, will also be denoted by $\langle$,$\rangle . By C^{\infty}(N(M))$ we denote the $C^{\infty}$ sections from $M$ into $N(M)$.

Define the tensor

$$
\mathscr{R}^{\perp}: C^{\infty}(N(M))^{3} \rightarrow C^{\infty}(N(M))
$$

by putting

$$
\mathscr{R}_{p}^{\perp}\left(\xi_{1}, \xi_{2}\right) \xi_{3}=\sum_{j=1}^{n} R_{p}^{\perp}\left(A_{\xi_{1}}\left(e_{j}\right), A_{\xi_{2}}\left(e_{j}\right)\right) \xi_{3},
$$

$p \in M, \xi_{1}, \xi_{2}, \xi_{3} \in N(M)_{p}$; where $A$ is the shape operator, $R^{\perp}$ is the curvature operator of the normal connection $\nabla^{\perp}$ and $\left\{e_{1}, \ldots, e_{n}\right\}$ is an aribitrary orthonormal basis of $T_{p} M$.

The above tensor was just defined in [O-S].

Given an Euclidean space $\mathbf{V}$ we will denote by $\mathscr{A}(\mathbf{V})$ the vector space of skew-symmetric endomorphisms of $\mathbf{V}$, with the usual inner product $($,$) , i.e.,$ $(A, B)=-\operatorname{trace}(A \circ B)$.

Lemma 2.1. Assume the hypothesis and notation of this section. Then, for all $\xi_{1}, \xi_{2}, \xi_{3}, \xi_{4} \in C^{\infty}(N(M))$, the following are verified:

(i) $\mathscr{R}^{\perp}\left(\xi_{1}, \xi_{2}\right)=-\mathscr{R}^{\perp}\left(\xi_{2}, \xi_{1}\right)$,

(ii) $\mathscr{R}^{\perp}\left(\xi_{1}, \xi_{2}\right) \xi_{3}+\mathscr{R}^{\perp}\left(\xi_{2}, \xi_{3}\right) \xi_{1}+\mathscr{R}^{\perp}\left(\xi_{3}, \xi_{1}\right) \xi_{2}=0$,

(iii) $\left\langle\mathscr{R}^{\perp}\left(\xi_{1}, \xi_{2}\right) \xi_{3}, \xi_{4}\right\rangle=-\left\langle\xi_{3}, \mathscr{R}^{\perp}\left(\xi_{1}, \xi_{2}\right) \xi_{4}\right\rangle$,

(iv) $\left\langle\mathscr{R}^{\perp}\left(\xi_{1}, \xi_{2}\right) \xi_{3}, \xi_{4}\right\rangle=\left\langle\mathscr{R}^{\perp}\left(\xi_{3}, \xi_{4}\right) \xi_{1}, \xi_{2}\right\rangle=-\frac{1}{2}\left(\left[A_{\xi_{1}}, A_{\xi_{2}}\right],\left[A_{\xi_{3}}, A_{\xi_{4}}\right]\right)$.

Proof. The proof was given in [O-S], but we reproduce it completely. Let $p \in M$ and let $\left\{e_{1}, \ldots, e_{n}\right\}$ be an orthonormal basis of $T_{p} M$.

$$
\begin{aligned}
\left\langle\mathscr{R}_{p}^{\perp}\left(\xi_{1}, \xi_{2}\right) \xi_{3}, \xi_{4}\right\rangle & =\left\langle\sum_{j=1}^{n} R_{p}^{\perp}\left(A_{\xi_{1}}\left(e_{j}\right), A_{\xi_{2}}\left(e_{j}\right)\right) \xi_{3}, \xi_{4}\right\rangle \\
& =\sum_{j=1}^{n}\left\langle\left[A_{\xi_{3}}, A_{\xi_{4}}\right]\left(A_{\xi_{1}}\left(e_{j}\right)\right), A_{\xi_{2}}\left(e_{j}\right)\right\rangle
\end{aligned}
$$

by the well-known formula, when the ambient space is of constant curvature,

$$
\begin{aligned}
= & \sum_{j=1}^{n}\left\langle A_{\xi_{2}} \circ\left[A_{\xi_{3}}, A_{\xi_{4}}\right] \circ A_{\xi_{1}}\left(e_{j}\right), e_{j}\right\rangle \\
= & \operatorname{trace}_{p}\left(A_{\xi_{2}} \circ\left[A_{\xi_{3}}, A_{\xi_{4}}\right] \circ A_{\xi_{1}}\right) \\
= & \frac{1}{2} \operatorname{trace}_{p}\left(A_{\xi_{2}} \circ\left[A_{\xi_{3}}, A_{\xi_{4}}\right] \circ A_{\xi_{1}}\right) \\
& +\frac{1}{2} \operatorname{trace}_{p}\left(\left(A_{\xi_{2}} \circ\left[A_{\xi_{3}}, A_{\xi_{4}}\right] \circ A_{\xi_{1}}\right)^{t}\right)
\end{aligned}
$$




$$
\begin{aligned}
= & \frac{1}{2} \operatorname{trace}_{p}\left(A_{\xi_{1}} \circ A_{\xi_{2}} \circ\left[A_{\xi_{3}}, A_{\xi_{4}}\right]\right) \\
& -\frac{1}{2} \operatorname{trace}_{p}\left(A_{\xi_{1}} \circ\left[A_{\xi_{3}}, A_{\xi_{4}}\right] \circ A_{\xi_{2}}\right) \\
= & \frac{1}{2} \operatorname{trace}_{p}\left\{A_{\xi_{1}} \circ A_{\xi_{2}} \circ\left[A_{\xi_{3}}, A_{\xi_{4}}\right]-A_{\xi_{2}} \circ A_{\xi_{1}} \circ\left[A_{\xi_{3}}, A_{\xi_{3}}\right]\right\} \\
= & \frac{1}{2} \operatorname{trace}_{p}\left(\left[A_{\xi_{1}}, A_{\xi_{2}}\right] \circ\left[A_{\xi_{3}}, A_{\xi_{4}}\right]\right)
\end{aligned}
$$

which proves (i), (iii), and (iv).

As we have seen above,

$$
\begin{aligned}
\left\langle\mathscr{R}_{p}^{\perp}\left(\xi_{1}, \xi_{2}\right) \xi_{3}, \xi_{4}\right\rangle= & \operatorname{trace}_{p}\left(A_{\xi_{2}} \circ\left[A_{\xi_{3}}, A_{\xi_{4}}\right] \circ A_{\xi_{1}}\right) \\
= & \operatorname{trace}_{p}\left(A_{\xi_{2}} \circ A_{\xi_{3}} \circ A_{\xi_{4}} \circ A_{\xi_{1}}\right) \\
& -\operatorname{trace}_{p}\left(A_{\xi_{2}} \circ A_{\xi_{4}} \circ A_{\xi_{3}} \circ A_{\xi_{1}}\right) \\
= & \operatorname{trace}_{p}\left(A_{\xi_{1}} \circ A_{\xi_{2}} \circ A_{\xi_{3}} \circ A_{\xi_{4}}\right) \\
& -\operatorname{trace}_{p}\left(A_{\xi_{3}} \circ A_{\xi_{1}} \circ A_{\xi_{2}} \circ A_{\xi_{4}}\right) .
\end{aligned}
$$

Summing over all cyclic permutations of $\{1,2,3\}$ we clearly obtain (ii).

Observe that, from (iv), we have that $R_{p}^{\perp}=0 \Leftrightarrow \mathscr{R}_{p}^{\perp}=0$.

We have much more than this. The following result tells us that $\mathscr{R}^{\perp}$ carries the same geometrical information as $R^{\perp}$.

Proposition 2.2. Assume the notation and assumptions of this section. Then, for all $p \in M$, the linear space of skew-symmetric endomorphisms of $N(M)_{p}$ spanned by the set $\left\{R_{p}^{\perp}(X, Y): X, Y \in T_{p} M\right\}$ coincides with that spanned by the set $\left\{\mathscr{R}_{p}^{\perp}(\xi, \eta): \xi, \eta \in N(M)_{p}\right\}$.

In order to prove the last fact we shall next define $\mathscr{R}^{\perp}$ in an equivalent but convenient way.

First of all observe that, by (i) of Lemma 2.1, we can see, for each $p \in M$,

$$
\mathscr{R}_{p}^{\perp}: \Lambda^{2}\left(N(M)_{p}\right) \rightarrow \mathscr{A}\left(N(M)_{p}\right)
$$

by putting

Similarly we can see

$$
\mathscr{R}_{p}^{\perp}(\xi \wedge \eta)=\mathscr{R}_{p}^{\perp}(\xi, \eta)
$$

$$
R_{p}^{\perp}: \Lambda^{2}\left(T_{p} M\right) \rightarrow \mathscr{A}\left(N(M)_{p}\right) .
$$

Consider now

$$
\Lambda^{2}\left(N(M)_{p}\right) \stackrel{L_{p}}{\longrightarrow} \mathscr{A}\left(T_{p} M\right) \stackrel{h_{p}}{\longrightarrow} \Lambda^{2}\left(T_{p} M\right) \stackrel{R_{p}^{\perp}}{\longrightarrow} \mathscr{A}\left(N(M)_{p}\right),
$$

where $L_{p}(\xi \wedge \eta)=\left[A_{\xi}, A_{\eta}\right]$ and $h_{p}$ is the isomorphism given by

$$
\left\langle h_{p}^{-1}(x \wedge y)(u), v\right\rangle=\langle x, u\rangle \cdot\langle y, v\rangle-\langle y, u\rangle \cdot\langle x, v\rangle .
$$


By a straightforward calculation we have

Lemma 2.3. $\mathscr{R}_{p}^{\perp}=-R_{p}^{\perp} \circ h_{p} \circ L_{p}$.

Put on $\Lambda^{2}\left(T_{p} M\right)$ the inner product defined by

$$
((v, w))=\left(h_{p}^{-1}(v), h_{p}^{-1}(w)\right)=-\operatorname{trace}\left(h_{p}^{-1}(v) \circ h_{p}^{-1}(w)\right) .
$$

Then we have

Lemma 2.4. $\operatorname{ker} R_{p}^{\perp}=\left(h_{p} \circ L_{p}\left(\Lambda^{2}\left(N(M)_{p}\right)\right)\right)^{\perp}$.

Proof. Let $\left\{e_{1}, \ldots, e_{n}\right\}$ be an orthonormal basis of $T_{p} M$, and let $u=$ $\sum_{k<l} a_{k l} \cdot e_{k} \wedge e_{l}=\Lambda^{2}\left(T_{p} M\right)$. If $\xi, \eta \in N(M)_{p}$ are arbitrary,

$$
\begin{aligned}
\left\langle R_{p}^{\perp}(u) \xi, \eta\right\rangle & =\left\langle\sum_{k<l} a_{k l} \cdot R_{p}^{\perp}\left(e_{k}, e_{l}\right) \xi, \eta\right\rangle \\
& =\sum_{k<l} a_{k l} \cdot\left\langle\left[A_{\xi}, A_{\eta}\right]\left(e_{k}\right), e_{l}\right\rangle
\end{aligned}
$$

but

$$
\begin{aligned}
\left(\left(u, h_{p} \circ L_{p}(\xi \wedge \eta)\right)\right) & =-\operatorname{trace}\left(h_{p}^{-1}(u) \circ L_{p}(\xi \wedge \eta)\right) \\
& =\sum_{s, t}\left\langle h_{p}^{-1}(u)\left(e_{s}\right), e_{t}\right\rangle \cdot\left\langle\left[A_{\xi}, A_{\eta}\right]\left(e_{s}\right), e_{t}\right\rangle \\
& =2 \cdot \sum_{k<l} a_{k l}\left\langle\left[A_{\xi}, A_{\eta}\right]\left(e_{k}\right), e_{l}\right\rangle,
\end{aligned}
$$

then

$$
\left\langle R_{p}^{\perp}(u) \xi, \eta\right\rangle=\frac{1}{2} \cdot\left(\left(u, h_{p} \circ L_{p}(\xi \wedge \eta)\right)\right),
$$

which clearly implies the Lemma.

Proof of the proposition. It is immediate from Lemma 2.4.

\section{The MAIN Result}

Theorem 3.1. Let $M^{n}$ be an immersed submanifold of a Riemannian manifold $Q^{N}$ of constant curvature. Let $p \in M$ and let $\Phi^{*}$ be the restricted holonomy group of the normal connection at $p$. Then $\Phi^{*}$ is compact, there exists a unique (up to order) orthogonal decomposition of the normal space at $p, N(M)_{p}=$ $\mathbf{V}_{0} \oplus \cdots \oplus \mathbf{V}_{k}$, into $\Phi^{*}$-invariant subspaces, and there exist $\Phi_{0}, \ldots, \Phi_{k}$ normal Lie subgroups of $\Phi^{*}$ such that:

(i) $\Phi^{*}=\Phi_{0} \times \cdots \times \Phi_{k}$ (direct product).

(ii) $\Phi_{i}$ acts trivially on $\mathbf{V}_{j}$ if $i \neq j$.

(iii) $\Phi_{0}=\{1\}$ and, if $i \geq 1, \Phi_{i}$ acts irreducibly on $\mathbf{V}_{i}$ as the isotropy representation of a simple Riemannian symmetric space.

We keep the notation and assumptions of $\S 2$. 
Let $p \in M$ be fixed, and let $\gamma:[0,1] \rightarrow M$ be a piecewise differentiable curve with $\gamma(1)=p$. Denote by $\gamma^{*}\left(\mathscr{R}^{\perp}\right)$ the tensor of type $(1,3)$ in $N(M)_{p}$ defined by

$$
\gamma^{*}\left(\mathscr{R}^{\perp}\right)(v, w) z=P_{\gamma}\left(\mathscr{R}_{q}^{\perp}\left(P_{\gamma}^{-1}(v), P_{\gamma}^{-1}(w)\right) P_{\gamma}^{-1}(z)\right),
$$

where $\gamma(0)=q$ and $P_{\gamma}$ denotes the parallel displacement along $\gamma$ with the normal connection.

Denote by $\mathscr{S}$ the linear subspace of the tensors of type $(1,3)$ of $N(M)_{p}$, generated by all the $\gamma^{*}\left(\mathscr{R}^{\perp}\right)$, where $\gamma$ runs over all piecewise differentiable curves ending at $p$.

From the theorem of Ambrose-Singer and Proposition 2.2 we have that the Lie algebra $g$ of the restricted normal holonomy group $\Phi^{*}$ at $p$ coincides with the linear span of the set $\left\{R(u, v): R \in \mathscr{S}, u, v \in N(M)_{p}\right\}$.

From Lemma 2.1, we have that if $R \in \mathscr{S}$, then

(i) $R(u, v)=-R(v, u)$,

(ii) $R(u, v) w+R(v, w) u+R(w, u) v=0$,

(iii) $\langle R(u, v) w, z\rangle=-\langle w, R(u, v) z\rangle$,

(iv) $\langle R(u, v) w, z\rangle=\langle R(w, z) u, v\rangle$.

Decompose orthogonally

$$
N(M)_{p}=\mathbf{V}_{0} \oplus \cdots \oplus \mathbf{V}_{k}
$$

into $\Phi^{*}$-invariant subspaces such that $\Phi^{*}$ acts trivially in $\mathbf{V}_{0}$ and, if $i \geq 1$, $\Phi^{*}$ acts irreducibly in $\mathbf{V}_{i}\left(\operatorname{dim} \mathbf{V}_{i} \geq 2\right)$.

If $u \in N(M)_{p}$, denote by $u_{i}$ the projection of $u$ into $\mathbf{V}_{i}$.

Lemma 3.2. Let $x, y \in N(M)_{p}$ and let $R \in \mathscr{S}$. Then

(i) $R\left(x_{i}, y_{j}\right)=0$ if $i \neq j$;

(ii) $R(x, y)=\sum_{i=0}^{k} R\left(x_{i}, y_{i}\right)$;

(iii) $R\left(x_{i}, y_{i}\right) \mathbf{V}_{j}=\{0\}$ if $i \neq j$;

(iv) $R\left(x_{i}, y_{i}\right) \mathbf{V}_{i} \subset \mathbf{V}_{i}$.

Proof. It is the same of that given in [S, pp. 217, 218], but as it is very easy we write it down.

If $i \neq j$ and $u, v \in N(M)_{p}$ then

$$
\left\langle R\left(x_{i}, y_{j}\right) u, v\right\rangle=\left\langle R(u, v) x_{i}, y_{j}\right\rangle=0
$$

because $R(u, v) \in g$ and $g$ leaves $\mathbf{V}_{i}$ invariant. This proves (i) and therefore (ii).

Let $v_{j} \in \mathbf{V}_{j}$, then

$$
R\left(x_{i}, y_{i}\right) v_{j}=-R\left(y_{i}, v_{j}\right) x_{i}-R\left(v_{j}, x_{i}\right) y_{i}=0
$$

by part (i), which proves (iii).

Part (iv) is immediate.

Let $g_{i}$ be the vector subspace of $g$ generated by all the $R\left(x_{i}, y_{i}\right), R \in \mathscr{S}$ and $x_{i}, y_{i} \in \mathbf{V}_{i}$. From the above lemma we easily derive (see [S, p. 218]). 


\section{Lemma 3.3.}

(i) $g_{0}=\{0\}$ and each $g_{i}$ is an ideal of $g$, for $i=0, \ldots, k$;

(ii) $g=g_{1} \oplus \cdots \oplus g_{k}$, with $\left[g_{i}, g_{j}\right]=\{0\}$ if $i \neq j$;

(iii) $g_{i} \mathbf{V}_{i}=\mathbf{V}_{i}$ for $i=0, \ldots, k$;

(iv) $g_{i} \mathbf{V}_{j}=\{0\}$ if $i \neq j$;

(v) $g_{i}$ acts irreducibly in $\mathbf{V}_{i}$, for $i=1, \ldots, k$.

Proof of Theroem 3.1. We keep the notation of this section. For $i=0, \ldots, k$, let $\Phi_{i}$ be the connected Lie subgroup of $\Phi^{*}$ (which is also connected) with Lie algebra $g_{i}$. Lemma 3.3 implies that we have the direct product $\Phi^{*}=$ $\Phi_{0} \times \cdots \times \Phi_{k}$, that $\Phi_{i}$ acts trivially on $\mathbf{V}_{j}$ if $i \neq j$ and that $\Phi_{i}$ acts irreducibly on $\mathbf{V}_{i}$ if $i \geq 1$. The uniqueness part of the theorem is now clear.

Now, a connected Lie subgroup of orthogonal transformations of a vector space which acts irreducibly on it must be compact (see [K-N, appendix 5]). Then each $\Phi_{i}$ is compact, and therefore $\Phi^{*}$ is compact.

Now, for $i \geq 1$, choose $R_{i} \in \mathscr{S}$ such that $R_{i}$ is not identically zero in $\mathbf{V}_{i}^{3}$. Each $\left[\mathbf{V}_{i}, R_{i}, \Phi_{i}\right]$ is an irreducible holonomy system, in the notation of [S]. Using [S, Theorem 5], we finish the proof, since, by Lemma 2.1(iv), $R_{i}$ must have negative scalar curvature.

In a future paper we will use the above results to establish the relation between isoparametric submanifolds and the sense of Terng and those in the sense of Strübing.

\section{ACKNOWLEDGMENTS}

The author would like to thank Professors Abdus Salam, J. Eells, and A. Verjovsky of the I.C.T.P. for their hospitality.

\section{REFERENCES}

[B] M. Berger, Sur les groupes d'holonomie homogène des variétés à connexion affine et des variétés Riemannienes, Bull. Soc. Math. France 83 (1955), 279, 230.

[K-N] S. Kobayashi and K. Nomizu, Foundations of differential geometry, vol. I, Interscience Publishers, 1963.

[S] J. Simons, On the transitivity of holonomy systems, Ann. of Math. (2) 76 (1962).

[S-O] C. Olmos and C. Sanchez, A geometric characterization of $R$-spaces, preprint.

Mathematics Section, International Centre for Theoretical Physics, P.O. Box 586, 34100, Trieste, ItALY 